PACIFIC JOURNAL OF MATHEMATICS

Vol. 178, No. 2, 1997

\title{
GLOBAL HEAT KERNEL ESTIMATES
}

\author{
JIAPING WANG
}

\begin{abstract}
In this paper, by first deriving a global version of gradient estimates, we obtain both upper and lower bound estimates for the heat kernel satisfying Neumann boundary conditions on a compact Riemannian manifold with nonconvex boundary.
\end{abstract}

\section{Introduction.}

Let $M$ be a compact Riemannian manifold with boundary $\partial M$. In their fundamental work $[\mathbf{L}-\mathbf{Y}]$, P. Li and S.T. Yau had derived a version of gradient estimates for the positive solutions to the heat equations on $M$. Using those estimates, they then deduced a Harnack type inequality and demonstrated how that be applied to establish various upper and lower heat kernel bounds away from the boundary for both the Dirichlet and Neumann boundary conditions. Due to the interior nature of their gradient estimates, in general the heat kernel bounds do not extend up to the boundary. However, when the boundary is convex or the manifold is closed, the gradient estimates are valid globally, and so are the corresponding heat kernel bounds. In fact, in this case the upper and lower heat kernel bounds they obtained are sharp when the Ricci curvature of the manifold is nonnegative. One of our purposes in this paper is to demonstrate that a global version of their gradient estimates is also available when the boundary of $M$ is nonconvex. This enables us to prove a global Harnack type inequality, with which one conveniently obtains an upper bound estimate valid up to the boundary for the heat kernel of $M$ satisfying Neumann boundary conditions. As an application, we show that it together with a result of Varopoulos $[\mathbf{V}]$ readily gives us an estimate of the Neumann Sobolev constant of a general compact manifold with nonconvex boundary. Turning around, we then derive a lower bound estimate for the heat kernel. Notice that Croke $[\mathbf{C r}]$ has estimated the isoperimetric constants for the closed manifolds in terms of various geometric data. The approach taken here does not allow us to estimate the isoperimetric constants.

The method employed here to estalish the gradient estimate essentially follows from $[\mathbf{L}-\mathbf{Y}]$. However, we shall point out that there are some technical 
complications due to the nonconvexity of the boundary as the estimates then necessarily involve the second fundamental form of $\partial M$ and a so-called "interior rolling ball condition" for the boundary $\partial M$. The interior rolling ball condition was used by $\mathrm{R}$. Chen in $[\mathbf{C}]$ to give an estimate of the first nonzero Neumann eigenvalue of $M$. We would also like to point out that the argument here can be easily adapted to more general cases, for example, the Schrödinger operators as considered in $[\mathbf{L}-\mathbf{Y}]$. But here we confine ourselves only to consider the heat operators.

Another problem that we want to consider in this paper is to establish the equivalence between the validity of a version of parabolic Harnack inequality and the existence of comparable upper and lower Gaussian bounds on the minimal heat kernel on a general complete manifold. It had been proved independently by Grigor'yan $[\mathbf{G}]$ and Saloff-Coste $[\mathbf{S C}]$ that a type of parabolic Harnack inequality is characterized by the volume doubling property and the weak-Neumann Poincaré inequality. On the other hand, it is not difficult to see from the argument in $[\mathbf{L}-\mathbf{Y}]$ that the validity of such a parabolic Harnack inequality implies a Gaussian upper bound for the minimal heat kernel. Here, we first notice that such a Gaussian upper bound together with the parabolic Harnack inequality actually implies that there exists a comparable lower bound for the heat kernel. Then, with the help of a result by Fabes and Stroock $[\mathbf{F}-\mathbf{S}]$ and the above mentioned characterization of the parabolic Harnack inequality, we show that the existence of such upper and lower bounds on the heat kernel actually implies that the parabolic Harnack inequality holds. As a consequence, one sees that the existence of such comparable upper and lower Gaussian bounds for the heat kernel is an invariant property under quasi-isometries on the manifold.

The paper is organized as follows. In Section 2, we derive the global gradient estimates for the positive solutions to the heat equation on a compact manifold with boundary and satisfying the Neumann boundary conditions. In Section 3, using the result from Section 2, we establish an upper bound for the heat kernel of a compact manifold which satisfies Neumann boundary conditions and as an application give an estimate of the Neumann Sobolev constant. Finally, in Section 4, we prove the equivalence between the validity of a type of parabolic Harnack inequality and the existence of Gaussian bounds on the heat kernel on a general complete manifold.

We would like to thank Professors Peter Li, Rick Schoen and Leon Simon for their interest and many helpful suggestions. 


\section{Global Gradient Estimates.}

Let $\left(M^{n}, g\right)$ be an $n$-dimensional compact Riemannian manifold with boundary $\partial M$. Let $\frac{\partial}{\partial \nu}$ be the outward pointing unit normal vector to $\partial M$, and denote the second fundamental form of $\partial M$ with respect to $\frac{\partial}{\partial \nu}$ by II. Our goal in this section is to derive estimates on the derivatives of positive solutions $u(x, t)$ on $M \times(0, \infty)$ of the equation

$$
\left\{\begin{array}{l}
\left(\Delta-\frac{\partial}{\partial t}\right) u(x, t)=0 \\
\left.\frac{\partial u}{\partial \nu}\right|_{\partial M}=0 .
\end{array}\right.
$$

Our estimates are of global nature and will be valid up to the boundary of $M$. The corresponding interior estimates were previously established by $\mathrm{P}$. Li and S.T. Yau in their fundamental work $[\mathbf{L}-\mathbf{Y}]$. In fact, we shall use the same method developed by them in $[\mathbf{L}-\mathbf{Y}]$.

Definition 2.1. $\partial M$ is said to satisfy the "interior rolling R-ball" condition if for each point $p \in \partial M$ there is a geodesic ball $B_{q}\left(\frac{R}{2}\right)$, centered at $q \in M$ with radius $\frac{R}{2}$, such that $p=B_{q}\left(\frac{R}{2}\right) \cap \partial M$ and $B_{q}\left(\frac{R}{2}\right) \subset M$.

Theorem 2.2. Let $\left(M^{n}, g\right)$ be a compact Riemannian manifold with boundary $\partial M$. Suppose that $\partial M$ satisfies the "interior rolling $R$-ball" condition. Let $K$ and $H$ be nonnegative constants such that the Ricci curvature $\operatorname{Ric}_{M}$ of $M$ is bounded below by $-K$ and the second fundamental form II of $\partial M$ is bounded below by $-H$. By choosing $R$ small, we have for any positive solution $u(x, t)$ of (2.1) on $M \times(0, \infty)$,

$$
\frac{|\nabla u|^{2}}{u^{2}}-\alpha \frac{u_{t}}{u} \leq C_{1}+\frac{C_{2}}{t}
$$

on $M \times(0, \infty)$ for all constant

$$
\alpha>(H+1)^{2} \text { and } 0<\beta<\frac{1}{2}
$$

where

$$
\begin{aligned}
C_{1} & =\frac{6 n \alpha(\alpha-1)(1+H)^{7} K}{\left(\alpha-(1+H)^{2}\right)^{2}}+\frac{309 n^{2} \alpha^{3}(\alpha-1)(1+H)^{10} H}{\left(\alpha-(1+H)^{2}\right)^{4} R^{2} \beta} \\
C_{2} & =\frac{n \alpha^{2}(\alpha-1)^{2}(1+H)^{4}}{(2-\beta)(1-\beta)\left(\alpha-(1+H)^{2}\right)^{2}} .
\end{aligned}
$$

Proof. Following [C], we define a function on $M$ by $\phi(x)=\psi\left(\frac{r(x)}{R}\right)$, where $r(x)$ denotes the distance from $x \in M$ to $\partial M$ and $\psi(r)$ is a nonnegative 
$C^{2}$-function defined on $[0, \infty)$ such that

$$
\left\{\begin{array}{l}
\psi(r) \leq H \text { if } r \in[0,1 / 2) \\
\psi(r)=H \text { if } r \in[1, \infty)
\end{array}\right.
$$

with $\psi(0)=0, \quad 0 \leq \psi^{\prime}(r) \leq 2 H, \quad \psi^{\prime}(0)=H$ and $\psi^{\prime \prime}(r) \geq-H$. Let $f=\log u$. Then $\left(\Delta-\frac{\partial}{\partial t}\right) f(x, t)=-|\nabla f|^{2}(x, t)$. Consider for every $\varepsilon>0$

$$
F(x, t)=t\left\{(1+\phi(x))^{2}\left(|\nabla f|^{2}(x, t)+\varepsilon\right)-\alpha f_{t}(x, t)\right\} .
$$

For any fixed $T<\infty$, since $F(x, t)$ is continuous on $\bar{M} \times[0, T]$, there exists $\left(p, t_{0}\right) \in \bar{M} \times[0, T]$ at which $F$ achieves its maximum. We may assume that $F\left(p, t_{0}\right)>0$ as otherwise (2.2) follows trivially.

Claim 1. $p \in \bar{M} \backslash \partial M$.

In fact, if $p \in \partial M$, then $\frac{\partial F}{\partial \nu}\left(p, t_{0}\right) \geq 0$. Let $e_{1}, e_{2}, \ldots, e_{n}$ be an orthonormal frame at $p$ with $e_{n}=\nu$. Notice that $f_{n}=f_{\nu}=\frac{\frac{\partial u}{\partial \nu}}{u}=0$ on $\partial M$. Therefore, denoting $\varphi(x)=(1+\phi(x))^{2}$, we have

$$
0 \leq \frac{\partial F}{\partial \nu}\left(p, t_{0}\right)=t_{0}\left(\frac{\partial \varphi}{\partial \nu}\left(|\nabla f|^{2}+\varepsilon\right)+2 \varphi \sum_{i=1}^{n} f_{i} f_{i \nu}-\alpha f_{\nu t}\right)\left(p, t_{0}\right)
$$

Since $f_{\nu}=0$ on $\partial M$ and $t_{0}>0$, we conclude that $\frac{\partial \varphi}{\partial \nu} \cdot \frac{1}{\varphi}+\frac{2 \sum_{i=1}^{n-1} f_{i} f_{i \nu}}{|\nabla f|^{2}+\varepsilon} \geq 0$ at $\left(p, t_{0}\right)$. By a direct computation, one shows that

$$
\sum_{i=1}^{n-1} f_{i} f_{i \nu}=-\mathrm{II}(\nabla f, \nabla f) \leq H|\nabla f|^{2} .
$$

Thus at $\left(p, t_{0}\right)$, if we choose $R<1$, then

$$
\begin{aligned}
& \frac{\partial \varphi}{\partial \nu} \frac{1}{\varphi}+\frac{2 \sum_{i=1}^{n-1} f_{i} f_{i \nu}}{|\nabla f|^{2}+\varepsilon} \\
& \leq-\frac{2 H}{R}+\frac{2 H|\nabla f|^{2}}{|\nabla f|^{2}+\varepsilon}<0 .
\end{aligned}
$$

This is a contradiction and the claim follows. Thus, $F(x, t)$ achieves its maximum at $\left(p, t_{0}\right) \in(\bar{M} \backslash \partial M) \times(0, T]$. 
Hence at $\left(p, t_{0}\right), \nabla F=0, \frac{\partial F}{\partial t} \geq 0$ and $\Delta F \leq 0$. In the following, all the computations are performed at the point $\left(p, t_{0}\right)$ and the summation convention is used with indices $i$ and $j$ both moving between 1 and $n$. Direct computation gives us

$$
\begin{aligned}
\Delta F & =t\left(\Delta \varphi \cdot\left(|\nabla f|^{2}+\varepsilon\right)+\varphi \Delta|\nabla f|^{2}+2 \nabla \varphi \cdot \nabla|\nabla f|^{2}-\alpha(\Delta f)_{t}\right) \\
& =t\left(\Delta \varphi\left(|\nabla f|^{2}+\varepsilon\right)+2 \varphi\left(f_{i j}^{2}+f_{i} f_{i j j}\right)+2 \nabla \varphi \cdot \nabla|\nabla f|^{2}-\alpha(\Delta f)_{t}\right) .
\end{aligned}
$$

Since

$$
\begin{aligned}
f_{i} f_{i j j} & =f_{i} f_{j j i}+\operatorname{Ric}(\nabla f, \nabla f) \\
& \geq \nabla f \cdot \nabla(\Delta f)-K|\nabla f|^{2}
\end{aligned}
$$

and $\Delta f=f_{t}-|\nabla f|^{2}$, we obtain

$$
\begin{aligned}
\Delta F \geq & t\left\{\Delta \varphi\left(|\nabla f|^{2}+\varepsilon\right)+2 \varphi\left(f_{i j}^{2}-K|\nabla f|^{2}+\nabla f \cdot \nabla(\Delta f)\right)\right. \\
& \left.+2 \nabla \varphi \cdot \nabla|\nabla f|^{2}-\alpha\left(f_{t}-|\nabla f|^{2}\right)_{t}\right\} .
\end{aligned}
$$

Also $F_{t}=\varphi\left(|\nabla f|^{2}+\varepsilon\right)-\alpha f_{t}+t\left(\varphi|\nabla f|_{t}^{2}-\alpha f_{t t}\right)$. Thus $0 \geq \Delta F-\frac{\partial F}{\partial t}$ implies

$$
\begin{aligned}
0 \geq & t\left\{\Delta \varphi\left(|\nabla f|^{2}+\varepsilon\right)+2 \varphi f_{i j}^{2}-2 K \varphi|\nabla f|^{2}+2 \varphi \nabla f \cdot \nabla\left(f_{t}-|\nabla f|^{2}\right)\right. \\
& \left.+2 \nabla \varphi \cdot \nabla|\nabla f|^{2}+(\alpha-\varphi)|\nabla f|_{t}^{2}\right\}-\varphi\left(|\nabla f|^{2}+\varepsilon\right)+\alpha f_{t} \\
= & t\left\{\varepsilon \Delta \varphi+(\Delta \varphi-2 K \varphi)|\nabla f|^{2}+2 \varphi f_{i j}^{2}\right\}-\frac{F}{t} \\
& +t\left\{2 \nabla \varphi \cdot \nabla|\nabla f|^{2}-2 \varphi \nabla f \cdot \nabla|\nabla f|^{2}+2 \alpha \nabla f \cdot \nabla f_{t}\right\} .
\end{aligned}
$$

Using the fact that

$$
\nabla F=t\left\{\left(|\nabla f|^{2}+\varepsilon\right) \nabla \varphi+\varphi \nabla|\nabla f|^{2}-\alpha \nabla f_{t}\right\}=0,
$$

we get

$$
\begin{aligned}
0 \geq & t\left\{\varepsilon \Delta \varphi+(\Delta \varphi-2 K \varphi)|\nabla f|^{2}+2 \varphi f_{i j}^{2}\right\}-\frac{F}{t} \\
& +2 t \nabla \varphi \cdot \nabla|\nabla f|^{2}+2 t\left(|\nabla f|^{2}+\varepsilon\right) \nabla \varphi \cdot \nabla f
\end{aligned}
$$




$$
\begin{aligned}
\geq & t\left\{\varepsilon \Delta \varphi+(\Delta \varphi-2 K \varphi)|\nabla f|^{2}+2 \varphi f_{i j}^{2}\right\}-\frac{F}{t} \\
& +4 t \varphi_{i} f_{j} f_{i j}-2 t|\nabla \varphi||\nabla f|^{3}-2 t|\nabla \varphi||\nabla f| \varepsilon \\
\geq & 2 \varphi t f_{i j}^{2}+t(\Delta \varphi-2 K \varphi)|\nabla f|^{2}+\varepsilon t \Delta \varphi-\frac{F}{t} \\
& -\frac{4 t}{\beta}|\nabla \varphi|^{2}|\nabla f|^{2}-\beta t f_{i j}^{2}-2 t|\nabla \varphi||\nabla f|^{3}-|\nabla \varphi|^{2} t \varepsilon-|\nabla f|^{2} t \varepsilon \\
\geq & (2 \varphi-\beta) t f_{i j}^{2}+t\left(\Delta \varphi-2 K \varphi-\frac{4}{\beta}|\nabla \varphi|^{2}-\varepsilon\right)|\nabla f|^{2} \\
& -2 t|\nabla \varphi||\nabla f|^{3}+\varepsilon t\left(\Delta \varphi-|\nabla \varphi|^{2}\right)-\frac{F}{t} .
\end{aligned}
$$

Since $\sum f_{i j}^{2} \geq \sum f_{i i}^{2} \geq \frac{\left(\sum f_{i i}\right)^{2}}{n}=\frac{(\Delta f)^{2}}{n}=\frac{\left(|\nabla f|^{2}-f_{t}\right)^{2}}{n}$,

$$
\begin{aligned}
0 \geq & \frac{(2 \varphi-\beta) t^{2}}{n}\left(|\nabla f|^{2}-f_{t}\right)^{2}-2 t^{2}|\nabla \varphi||\nabla f|^{3} \\
& +t^{2}\left(\Delta \varphi-2 K \varphi-\frac{4}{\beta}|\nabla \varphi|^{2}-\varepsilon\right)|\nabla f|^{2}+\varepsilon t^{2}\left(\Delta \varphi-|\nabla \varphi|^{2}\right)-F
\end{aligned}
$$

\section{Claim 2.}

$$
\left(|\nabla f|^{2}-f_{t}\right)^{2} \geq \frac{(1-\beta)\left(\alpha-(1+H)^{2}\right)^{2}}{(\alpha-1)^{2}(H+1)^{4}}\left(\varphi\left(|\nabla f|^{2}+\varepsilon\right)-f_{t}\right)^{2}-\frac{2 \varepsilon^{2}}{\beta} .
$$

In fact, using the elementary inequality $a^{2} \geq(1-\beta)(a+b)^{2}-\frac{2}{\beta} b^{2}$, we conclude that

$$
\left(|\nabla f|^{2}-f_{t}\right)^{2} \geq(1-\beta)\left(|\nabla f|^{2}+\varepsilon-f_{t}\right)^{2}-\frac{2 \varepsilon^{2}}{\beta} .
$$

On the other hand, $F \geq 0$ at $\left(p, t_{0}\right)$, hence

$$
\varphi\left(|\nabla f|^{2}+\varepsilon\right)-\alpha f_{t} \geq 0 .
$$

In other words,

$$
f_{t} \leq \frac{\varphi}{\alpha}\left(|\nabla f|^{2}+\varepsilon\right)
$$

Therefore

$$
(1-\beta)\left(|\nabla f|^{2}+\varepsilon-f_{t}\right)^{2}-\frac{(1-\beta)\left(\alpha-(1+H)^{2}\right)^{2}}{(\alpha-1)^{2}(H+1)^{4}}\left(\varphi\left(|\nabla f|^{2}+\varepsilon\right)-f_{t}\right)^{2}
$$




$$
\begin{aligned}
=(1 & -\beta)\left[\left(|\nabla f|^{2}+\varepsilon-f_{t}\right)+\frac{\alpha-(1+H)^{2}}{(\alpha-1)(H+1)^{2}}\left(\varphi\left(|\nabla f|^{2}+\varepsilon\right)-f_{t}\right)\right] \\
& \cdot\left[\left(|\nabla f|^{2}+\varepsilon-f_{t}\right)-\frac{\alpha-(1+H)^{2}}{(\alpha-1)(H+1)^{2}}\left(\varphi\left(|\nabla f|^{2}+\varepsilon\right)-f_{t}\right)\right] \\
=(1-\beta)\left[\left(1+\varphi \frac{\alpha-(1+H)^{2}}{(\alpha-1)(H+1)^{2}}\right)\left(|\nabla f|^{2}+\varepsilon\right)\right. & \\
& \left.-\left(1+\frac{\alpha-(1+H)^{2}}{(\alpha-1)(H+1)^{2}}\right) f_{t}\right] \\
& \cdot\left[\left(1-\varphi \frac{\alpha-(1+H)^{2}}{(\alpha-1)(H+1)^{2}}\right)\left(|\nabla f|^{2}+\varepsilon\right)-\left(1-\frac{\alpha-(1+H)^{2}}{(\alpha-1)(H+1)^{2}}\right) f_{t}\right] .
\end{aligned}
$$

Using (2.4), one easily checks that the above expression is nonnegative and the claim is verified. Using the claim and (2.3), we obtain

$$
\begin{aligned}
0 \geq & \frac{(2 \varphi-\beta) t^{2}}{n} \cdot \frac{(1-\beta)\left(\alpha-(1+H)^{2}\right)^{2}}{(\alpha-1)^{2}(1+H)^{4}}\left(\varphi\left(|\nabla f|^{2}+\varepsilon\right)-f_{t}\right)^{2} \\
& -2 t^{2}|\nabla \varphi||\nabla f|^{3}+t^{2}\left(\Delta \varphi-2 K \varphi-\frac{4}{\beta}|\nabla \varphi|^{2}-\varepsilon\right)|\nabla f|^{2} \\
& +\varepsilon t^{2}\left(\Delta \varphi-|\nabla \varphi|^{2}-\frac{2 \varepsilon(2 \varphi-\beta)}{n \beta}\right)-F
\end{aligned}
$$

Let $y=\varphi\left(|\nabla f|^{2}+\varepsilon\right)$ and $z=f_{t}$. Then

$$
\begin{aligned}
(y-z)^{2} & =\left[\frac{1}{\alpha}(y-\alpha z)+\frac{\alpha-1}{\alpha} y\right]^{2} \\
& =\frac{1}{\alpha^{2}}(y-\alpha z)^{2}+\left(\frac{\alpha-1}{\alpha}\right)^{2} y^{2}+\frac{2(\alpha-1)}{\alpha^{2}} y(y-\alpha z) \\
& \geq \frac{1}{\alpha^{2} t^{2}} F^{2}+\left(\frac{\alpha-1}{\alpha}\right)^{2} y^{2} \quad \text { as } y-\alpha z=\frac{F}{t}>0 .
\end{aligned}
$$

Combining (2.5) and (2.6), we get

$$
\begin{aligned}
0 \geq & \frac{(2 \varphi-\beta)(1-\beta)\left(\alpha-(1+H)^{2}\right)^{2}}{n \alpha^{2}(\alpha-1)^{2}(1+H)^{4}} F^{2}-F \\
& +\frac{(2 \varphi-\beta)(1-\beta) t^{2}\left(\alpha-(1+H)^{2}\right)^{2}}{n \alpha^{2}(1+H)^{4}} y^{2}-2 t^{2}|\nabla \varphi||\nabla f|^{3} \\
& +t^{2}\left(\Delta \varphi-2 K \varphi-\frac{4}{\beta}|\nabla \varphi|^{2}-\varepsilon\right)|\nabla f|^{2}
\end{aligned}
$$




$$
+\varepsilon t^{2}\left(\Delta \varphi-|\nabla \varphi|^{2}-\frac{2 \varepsilon(2 \varphi-\beta)}{n \beta}\right) .
$$

By $[\mathbf{C}]$, if we choose $R$ sufficiently small, then

$$
\Delta \phi \geq-\frac{2(n-1) H(2 H+1)}{R}-\frac{H}{R^{2}} .
$$

Therefore,

$$
\begin{aligned}
\Delta \varphi & =2(1+\phi) \Delta \phi+2|\nabla \phi|^{2} \\
& \geq 2(1+H)\left(-\frac{2(n-1) H(3 H+1)}{R}-\frac{H}{R^{2}}\right) \\
& =-C_{3} .
\end{aligned}
$$

Hence

$$
\begin{aligned}
& \frac{(2 \varphi-\beta)(1-\beta)\left(\alpha-(1+H)^{2}\right)^{2}}{n \alpha^{2}(1+H)^{4}} y^{2}-2|\nabla \varphi||\nabla f|^{3} \\
& \quad+\left(\Delta \varphi-2 K \varphi-\frac{4}{\beta}|\nabla \varphi|^{2}-\varepsilon\right)|\nabla f|^{2} \\
& \geq \frac{\left(\alpha-(1+H)^{2}\right)^{2}}{2 n \alpha^{2}(1+H)^{4}} y^{2}-8 H(H+1) y^{\frac{3}{2}}-\left(C_{3}+2 K(1+H)^{2}\right. \\
& \left.\quad+\frac{64}{\beta} H^{2}(H+1)^{2}+\varepsilon\right) y .
\end{aligned}
$$

Consider $A y^{2}-B y^{\frac{3}{2}}-C y$, where $A, B, C$ all are positive. Clearly

$$
\begin{aligned}
& A y^{2}-B y^{\frac{3}{2}}-C y=\frac{A}{2} y^{2}+\frac{A}{2} y^{2}-B y^{\frac{3}{2}}+\frac{B^{2}}{2 A} y-\left(C+\frac{B^{2}}{2 A}\right) y \\
& \geq \frac{A}{2} y^{2}-\left(C+\frac{B^{2}}{2 A}\right) y=\frac{A}{2} y^{2}-\left(C+\frac{B^{2}}{2 A}\right) y+D^{2}-D^{2} \\
& \geq-D^{2}, \quad \text { where } D^{2}=\frac{\left(C+\frac{B^{2}}{2 A}\right)^{2}}{2 A} .
\end{aligned}
$$

Applying (2.9) to (2.8), we conclude from (2.7) that

$$
0 \geq \frac{(2 \varphi-\beta)(1-\beta)\left(\alpha-(1+H)^{2}\right)^{2}}{n \alpha^{2}(\alpha-1)^{2}(1+H)^{4}} F^{2}-F
$$




$$
+\varepsilon t^{2}\left(\Delta \varphi-|\nabla \varphi|^{2}-\frac{2 \varepsilon(2 \varphi-\beta)}{n \beta}\right)-D^{2} t^{2}
$$

where $D^{2}=\frac{\left(C+\frac{B^{2}}{2 A}\right)^{2}}{2 A}$, and $A=\frac{\left(\alpha-(1+H)^{2}\right)^{2}}{2 n \alpha^{2}(1+H)^{4}}, B=8 H(H+1)$ and $C=\left(C_{3}+2 K(1+H)^{2}+\frac{64}{\beta} H^{2}(H+1)^{2}+\varepsilon\right)$. From (2.10), one easily obtains

$$
F \leq \frac{1+\sqrt{1+4 P Q}}{2 P}
$$

where

$$
P=\frac{(2 \varphi-\beta)(1-\beta)\left(\alpha-(1+H)^{2}\right)^{2}}{n \alpha^{2}(\alpha-1)^{2}(1+H)^{4}}
$$

and

$$
Q=D^{2} t^{2}-\varepsilon t^{2}\left(\Delta \varphi-|\nabla \varphi|^{2}-\frac{2 \varepsilon(2 \varphi-\beta)}{n \beta}\right)
$$

But

$$
|\nabla f|^{2}-\alpha f_{t} \leq \varphi\left(|\nabla f|^{2}+\varepsilon\right)-\alpha f_{t}=\frac{F}{t} .
$$

Thus by (2.11) and letting $\varepsilon \rightarrow 0$, we have

$$
\begin{aligned}
|\nabla f|^{2}-\alpha f_{t} \leq & n \alpha^{2}(\alpha-1)^{2}(1+H)^{4} \\
& \cdot \frac{1+\sqrt{1+8 D^{2} t^{2}\left(\alpha-(1+H)^{2}\right)^{2} / n \alpha^{2}(\alpha-1)^{2}(1+H)^{2}}}{2(2 \varphi-\beta)(1-\beta)\left(\alpha-(1+H)^{2}\right)^{2} t} \\
\leq & \frac{n \alpha^{2}(\alpha-1)^{2}(1+H)^{4}\left(1+1+\frac{3 D t\left(\alpha-(1+H)^{2}\right)}{\sqrt{n} \alpha(\alpha-1)(1+H)}\right)}{2(2 \varphi-\beta)(1-\beta)\left(\alpha-(1+H)^{2}\right)^{2} t} .
\end{aligned}
$$

In conclusion,

$$
\frac{|\nabla u|^{2}}{u^{2}}-\alpha \frac{u_{t}}{u} \leq C_{1}+\frac{C_{2}}{t}
$$

where

$$
C_{1} \leq \frac{6 n \alpha(\alpha-1)(1+H)^{7} K}{\left(\alpha-\left(1+H^{2}\right)^{2}\right)^{2}}+\frac{309 n^{2} \alpha^{3}(\alpha-1)(1+H)^{10} H}{\left(\alpha-(1+H)^{2}\right)^{4} R^{2} \beta}
$$

and

$$
C_{2} \leq \frac{n \alpha^{2}(\alpha-1)^{2}(1+H)^{4}}{(2 \varphi-\beta)(1-\beta)\left(\alpha-(1+H)^{2}\right)^{2}} \leq \frac{n \alpha^{2}(\alpha-1)^{2}(1+H)^{4}}{(2-\beta)(1-\beta)\left(\alpha-(1+H)^{2}\right)^{2}} .
$$


The proof is completed.

Corollary 2.3 ([L-Y]). Let $M$ and $u$ be as in Theorem 2.2. If the boundary $\partial M$ of $M$ is convex, i.e., $H=0$, then for any $\alpha>1$,

$$
\frac{|\nabla u|^{2}}{u^{2}}-\alpha \frac{u_{t}}{u} \leq C_{3}+\frac{C_{4}}{t}
$$

where $C_{3}=\frac{6 n \alpha K}{\alpha-1}$ and $C_{4}=\frac{n \alpha^{2}}{2}$. If furthermore the Ricci curvature of $M$ is also nonnegative, then

$$
\frac{|\nabla u|^{2}}{u^{2}}-\frac{u_{t}}{u} \leq \frac{n}{2 t}
$$

Proof. In the first case that $H=0$, we use Theorem 2.2 and let $\beta$ approach to 0 . For the second case, since $K=0$, one can also let $\alpha$ approach to 1.

Remark. In our estimate, $R$ is chosen to be a positive constant less than 1 and is dependent on the upper bound of the sectional curvature of the manifold near the boundary. The upper bound of $R$ is explicitly determined by

$$
\sqrt{K_{R}} \tan \left(R \sqrt{K_{R}}\right) \leq \frac{H}{2}+\frac{1}{2}
$$

and

$$
\frac{H}{\sqrt{K_{R}}} \tan \left(R \sqrt{K_{R}}\right) \leq \frac{1}{2}
$$

where $K_{R}$ is the upper bound of the sectional curvature on the set $M_{R}=$ $\{x \in M \mid r(x) \leq R\}$ (see $[\mathbf{C}]$ ).

\section{Estimate of Sobolev Constants.}

In this section, we shall utilize the global gradient estimate in the previous section to derive an upper bound for the heat kernel satisfying Neumann boundary conditions on a general compact manifold with nonconvex boundary. The result is then used to estimate the Neumann Sobolev constants. Previously, Croke $[\mathbf{C r}]$ has obtained the estimates of Neumann isoperimetric constants for the manifolds without boundary. Our approach here does not allow us to estimate the isoperimetric constants. Thus, it is still left open to give an estimate of the isoperimetric constants for a compact manifold with nonconvex boundary. 
First, we establish the following Harnack inequality.

Theorem 3.1. Let $M$ be a compact manifold with boundary $\partial M$. Suppose that the Ricci curvature of $M$ satisfies $\operatorname{Ric}_{M} \geq-K, K \geq 0$ and the second fundamental form of $\partial M$ with respect to outward pointing normal $\nu$ satisfies $I I \geq-H, H \geq 0$. Suppose that $\partial M$ also satisfies the "interior rolling $R$ ball condition" with $R$ chosen small (see the remark at the end of Section 2). Then for any positive function $u(x, t)$ on $M \times(0, \infty)$ satisfying

$$
\begin{gathered}
\left\{\begin{array}{l}
\left(\Delta-\frac{\partial}{\partial t}\right) u=0 \\
\left.\frac{\partial u}{\partial \nu}\right|_{\partial M}=0,
\end{array}\right. \\
\begin{array}{c}
u\left(x_{1}, t_{1}\right) \leq u\left(x_{2}, t_{2}\right)\left(\frac{t_{2}}{t_{1}}\right)^{C_{5}} \exp \left(\frac{\alpha r^{2}\left(x_{1}, x_{2}\right)}{4\left(t_{2}-t_{1}\right)}+C_{6}\left(t_{2}-t_{1}\right)\right) \text { for any } \alpha> \\
(1+H)^{2} \text { and } 0<\beta<\frac{1}{2}, x_{1}, x_{2} \in M \text { and } 0<t_{1}<t_{2}<\infty, \text { where }
\end{array} \\
C_{5}=\frac{n \alpha(\alpha-1)^{2}(1+H)^{4}}{(2-\beta)(1-\beta)\left(\alpha-(1+H)^{2}\right)^{2}}, \\
C_{6}=\frac{6 n(\alpha-1)(1+H)^{7} K}{\left(\alpha-(1+H)^{2}\right)^{2}}+\frac{309 n^{2} \alpha^{2}(\alpha-1)(1+H)^{10} H}{\left(\alpha-(1+H)^{2}\right)^{4} R^{2} \beta} .
\end{gathered}
$$

Proof. The proof uses (2.2) and proceeds as Theorem 2.1 in $[\mathbf{L}-\mathbf{Y}]$. Hence it is omitted here.

Applying Theorem 3.1 and arguing as in [L-Y], one conveniently obtains an estimate of heat kernel of $M$ satisfying Neumann boundary condition.

Theorem 3.2. Let $M$ be as in Theorem 3.1. Let $H(x, y, t)$ be the heat kernel of $M$ satisfying Neumann boundary conditions. Then for any $\alpha>(1+H)^{2}$, $0<\beta<\frac{1}{2}$ and $\delta>0$, and $x, y$ on $M$,

$$
\begin{aligned}
& H(x, y, t) \\
& \leq(1+\delta)^{2 C_{5}} \exp \left(\frac{1+\alpha}{\delta}\right) V_{x}^{-\frac{1}{2}}(\sqrt{t}) V_{y}^{-\frac{1}{2}}(\sqrt{t}) \exp \left(-\frac{r^{2}(x, y)}{(4+\delta) t}+C_{6} \delta t\right),
\end{aligned}
$$

where $C_{5}$ and $C_{6}$ are given in Theorem 3.1.

In order to improve the above estimate for $t$ large, we use a method due to S.Y. Cheng and P. Li [C-L].

Lemma $3.3([\mathbf{C}-\mathbf{L}])$. Let $l_{1}(M)$ be the first nonzero eigenvalue of $M$ satisfying Neumann boundary condition, then for any fixed $t_{0}$ and all $t \geq t_{0}$,

$$
H(x, x, t) \leq \frac{1}{V(M)}+H\left(x, x, t_{0}\right) \exp \left(-l_{1}(M)\left(t-t_{0}\right)\right) .
$$


Proof. Let $\bar{H}(x, y, t)=H(x, y, t)-\frac{1}{V(M)}$. Then $\int_{M} \bar{H}(x, y, t) d y=0$. But by the semigroup property of $H(x, y, t)$, we have

$$
\begin{aligned}
\frac{\partial}{\partial t} \bar{H}(x, x, t) & =\frac{\partial}{\partial t} \int_{M} \bar{H}^{2}\left(x, y, \frac{t}{2}\right) d y \\
& =\int_{M} \bar{H}\left(x, y, \frac{t}{2}\right) \Delta \bar{H}\left(x, y, \frac{t}{2}\right) d y \\
& =-\int_{M}|\nabla \bar{H}|^{2}\left(x, y, \frac{t}{2}\right) d y \\
& \leq-l_{1}(M) \int_{M} \bar{H}^{2}\left(x, y, \frac{t}{2}\right) d y \\
& =-l_{1}(M) \bar{H}(x, x, t) .
\end{aligned}
$$

Integrating this differential inequality from $t_{0}$ to $t$ gives

$$
\bar{H}(x, x, t) \leq \bar{H}\left(x, x, t_{0}\right) \exp \left(-l_{1}(M)\left(t-t_{0}\right)\right) .
$$

Now the lemma follows using the definition of $\bar{H}(x, y, t)$.

If we choose $t_{0}=d^{2}$, where $d$ is the diameter of $M$, then by Lemma 3.3 and Theorem 3.2 together with the estimate of $l_{1}(M)$ obtained by $\mathrm{R}$. Chen in $[\mathbf{C}]$, we obtain the following estimate for the heat kernel of $M$ satisfying Neumann boundary conditions.

Theorem 3.4. Let $M$ be as in Theorem 3.1. Then for $t>0$ and $x, y$ in $M$,

$$
H(x, y, t) \leq \frac{1}{V(M)}+C_{7} V_{x}^{-\frac{1}{2}}(\sqrt{t}) V_{y}^{-\frac{1}{2}}(\sqrt{t}) \exp \left(-\frac{r^{2}(x, y)}{5 t}-C_{8} t\right),
$$

where $C_{7}$ and $C_{8}$ are two positive constants which only depend on $d, K, H$, $R$ and $n$ and can be explictly computed.

Finally, we want to use the above heat kernel bound to estimate the Neumann Sobolev constant of $M$. Consider the following type of Sobolev inequality

$$
\inf _{k \in \mathbf{R}}\left(\int_{M}|f-k|^{\frac{2 n}{n-2}}\right)^{\frac{n-2}{n}} \leq C(S) \int_{M}|\nabla f|^{2} \text { for all } f \in C^{\infty}(\bar{M}) .
$$

The minimum constant $C(S)$ is called the Neumann Sobolev constant of $M$. Using a result of Varopoulos $[\mathbf{V}]$, we can now estimate $C(S)$ from above in terms of geometric data of $M$. But before we do that, we need the following lemma. 
Lemma 3.5. Let $M$ be as in Theorem 3.1. Then $V_{x}(t) \geq C(d, K, H, V, R) t^{n}$ for some constant $C(d, K, H, V, R)>0$ and all $x \in M$ and $t \leq R$, where $V=V(M)$.

Proof. We first note that $M$ satisfies volume doubling property by Theorem 3.1 and 3.4 (see $[\mathrm{SC}]$ ).

Claim. For each $x \in M$ and $t \leq R$, there exists a geodesic ball $B_{p}\left(\frac{t}{6}\right) \subset$ $B_{x}(t)$ such that $B_{p}\left(\frac{t}{6}\right)$ does not intersect $\partial M$.

In fact, the claim is trivially true if the distance $d(x, \partial M)=r(x) \geq \frac{t}{2}$. Thus we consider the case $r(x)<\frac{t}{2} \leq \frac{R}{2}$. Let $y \in \partial M$ be such that $r(x, y)=r(x)$. Clearly, we have $B_{y}\left(\frac{t}{2}\right) \subset B_{x}(t)$. On the other hand, by the interior rolling R-ball condition, there exists a ball $B_{q}\left(\frac{R}{2}\right)$ such that it only intersects $\partial M$ at $y$. Now let $\gamma$ be a minimal geodesic connecting $y$ and $q$. Choose a point $p$ on $\gamma$ such that $r(p, y)=\frac{t}{4}$. Then it is easy to check that $B_{p}\left(\frac{t}{6}\right) \subset B_{y}\left(\frac{t}{2}\right) \subset B_{x}(t)$. Also, $B_{p}\left(\frac{t}{6}\right) \subset B_{q}\left(\frac{R}{2}\right)$. Since $r(p, y)>\frac{t}{6}, y$ is not in $B_{p}\left(\frac{t}{6}\right)$. Therefore $B_{p}\left(\frac{t}{6}\right)$ does not intersect $\partial M$ and the claim follows.

Now we divide the proof of the lemma into two cases.

Case (i): $r(x)<\frac{R}{2}$.

Let $M_{R}=\{x \in M: r(x) \leq R\}$. Then $B_{x}\left(\frac{t}{2}\right) \subset M_{R}$. By the claim, there exists $B_{p}\left(\frac{t}{12}\right) \subset B_{x}\left(\frac{t}{2}\right)$ and $B_{p}\left(\frac{t}{12}\right)$ has no intersection with $\partial M$. Since by the choice of $R, \sqrt{K_{R}} \tan \left(R \sqrt{K_{R}}\right) \leq \frac{H+1}{2}$, where $K_{R}$ is an upper bound of the sectional curvature on $M_{R}$. Therefore $K_{R} \leq \frac{H+1}{2 R}$. So the injectivity radius of the set $M_{R}$ can be estimated from below by a constant $C(V, R, H, d)$. In particular, we conclude that $V_{p}\left(\frac{t}{12}\right) \geq C(V, R, H, d) t^{n}$ for some constant $C(V, R, H, d)>0$. Thus we proved the lemma for this case.

Case (ii): $r(x) \geq \frac{R}{2}$.

In this case, we first show $V_{x}(r(x)) \geq C(V, R, H, d)$. In fact, arguing as in the claim, we conclude that there exists a ball $B_{p}\left(\frac{R}{12}\right) \subset M_{\frac{R}{3}}$ such that $B_{p}\left(\frac{R}{12}\right) \subset B_{x}(r(x))$. From case (i), we obtain $V_{x}(r(x)) \geq C(V, R, H, d)$. Now inside the ball $B_{x}(r(x))$ we may apply the Bishop volume comparison theorem. Notice that $r(x) \leq d$. We conclude that for $t \leq \frac{R}{2}$,

$$
\frac{V_{x}(r(x))}{V_{x}(t)} \leq \frac{V_{K}(r(x))}{V_{K}(t)} \leq \frac{V_{K}(d)}{V_{K}(t)}
$$

where $V_{K}(r)$ denotes the volume of the geodesic ball of radius $r$ in the space form of constant curvature $\frac{-K}{n-1}$. Putting together the preceding facts, we see that $V_{x}(t) \geq C(d, V, K, H, R) t^{n}$. The lemma is proved.

Combining Theorem 3.4 and Lemma 3.5, we obtain the following corollary. 
Corollary 3.6. Let $M$ be as in Theorem 3.1. Then for all $t>0$ and $x \in M$,

$$
H(x, x, t) \leq \frac{1}{V(M)}+A t^{-\frac{n}{2}}
$$

where $n=\operatorname{dim} M$ and $A=A(d, H, R, K, V)$ a positive constant which can be computed explicitly.

The following theorem is a consequence of a result in $[\mathbf{V}]$ and the preceding corollary.

Theorem 3.7. Let $M$ be as in Theorem 3.1. Then the Neumann Sobolev constant $C(S)$ of $M$ satisfies

$$
C(S) \leq C(n) A^{\frac{2}{n}}
$$

where $C(n)$ is a constant only depending on $n$ and $A$ is the constant in (3.12). In particular, $C(S) \leq C(d, H, R, K, V)$ a constant which can be explicitly computed.

Using Theorem 3.7, we now derive a lower bound estimate for the heat kernel $H(x, y, t)$. The following proof is adapted from $[\mathbf{C}-\mathbf{L}]$.

Theorem 3.8. Let $M$ be as in Theorem 3.1. Then the heat kernel $H(x, y, t)$ of $M$ satisfying the Neumann boundary conditions has estimates

$$
\frac{1}{V(M)}-C_{9} t^{-n / 2} \leq H(x, y, t) \leq \frac{1}{V(M)}+C_{10} t^{-n / 2}
$$

where $C_{9}$ and $C_{10}$ are two positive constants depending on $d, K, H, R$ and $V$.

Proof. Let $\bar{H}(x, y, t)=H(x, y, t)-\frac{1}{V(M)}$. Then arguing as in Lemma 3.3, we have

$$
\frac{\partial}{\partial t} \bar{H}(x, x, t)=-\int_{M}|\nabla \bar{H}|^{2}\left(x, y, \frac{t}{2}\right) d y .
$$

Since $\int_{M} \bar{H}(x, y, t) d y=0$, the Neumann Sobolev inequality gives

$$
\begin{aligned}
& C(S) \int_{M}|\nabla \bar{H}|^{2}\left(x, y, \frac{t}{2}\right) d y \geq\left(\int_{M}|\bar{H}|^{\frac{2 n}{n-2}}\left(x, y, \frac{t}{2}\right) d y\right)^{\frac{n-2}{n}} \\
& \geq\left(\int_{M}|\bar{H}|^{2}\left(x, y, \frac{t}{2}\right) d y\right)^{\frac{n+2}{n}}\left(\int_{M}|\bar{H}|\left(x, y, \frac{t}{2}\right) d y\right)^{-\frac{4}{n}} \\
& =(\bar{H}(x, x, t))^{\frac{n+2}{n}}\left(\int_{M}|\bar{H}|\left(x, y, \frac{t}{2}\right) d y\right)^{-\frac{4}{n}},
\end{aligned}
$$


where we have used the Hölder inequality. But

$$
\int_{M}|\bar{H}|\left(x, y, \frac{t}{2}\right) d y \leq \int_{M}\left(H\left(x, y, \frac{t}{2}\right)+\frac{1}{V(M)}\right) d y \leq 2 .
$$

Hence

$$
\int_{M}|\nabla \bar{H}|^{2}\left(x, y, \frac{t}{2}\right) d y \geq 2^{-\frac{4}{n}} C^{-1}(S)(\bar{H}(x, x, t))^{\frac{n+2}{n}} .
$$

In conclusion,

$$
\frac{\partial}{\partial t} \bar{H}(x, x, t) \leq-2^{-\frac{4}{n}} C^{-1}(S)(\bar{H}(x, x, t))^{\frac{n+2}{n}} .
$$

Integrating this differntial inequality from $\varepsilon>0$ to $t$ gives

$$
(\bar{H}(x, x, t))^{-\frac{2}{n}}-(\bar{H}(x, x, \varepsilon))^{-\frac{2}{n}} \geq \frac{2}{n}\left(2^{-\frac{4}{n}} C^{-1}(S)\right)(t-\varepsilon) .
$$

Letting $\varepsilon$ tend to 0 and noting that

$$
\lim _{\varepsilon \rightarrow 0}(\bar{H}(x, x, \varepsilon))^{-\frac{2}{n}}=0,
$$

we obtain

$$
\bar{H}(x, x, t) \leq c(n)(C(S))^{\frac{n}{2}} t^{-\frac{n}{2}} .
$$

Using the semi-group property of $\bar{H}(x, y, t)$, we conclude

$$
\begin{aligned}
|\bar{H}(x, y, 2 t)| & =\left|\int_{M} \bar{H}(x, z, t) \bar{H}(z, y, t) d z\right| \\
& \leq\left(\int_{M} \bar{H}^{2}(x, z, t) d z\right)^{\frac{1}{2}}\left(\int_{M} \bar{H}^{2}(z, y, t) d z\right)^{\frac{1}{2}} \\
& =\bar{H}^{\frac{1}{2}}(x, x, 2 t) \bar{H}^{\frac{1}{2}}(y, y, 2 t) .
\end{aligned}
$$

Therefore, putting the preceding inequalities together, we get

$$
|\bar{H}(x, y, t)| \leq c(n)(C(S))^{\frac{n}{2}} t^{-\frac{n}{2}} .
$$

Now the theorem follows from Theorem 3.7 and the definition of $\bar{H}(x, y, t)$.

Finally, we mention a corollary on estimating the Neumann eigenvalues. It can be easily proved by using the heat kernel estimates. 
Corollary 3.9. Let $M$ be as in Theorem 3.1. Then the $k$-th Neumann eigenvalue $\mu_{k}$ of $M$ satisfies

$$
\mu_{k} \geq C_{11}(K, H, V, R, d) k^{\frac{2}{n}}
$$

for all $k=0,1,2, \ldots$

\section{Parabolic Harnack Inequality.}

In this section, we turn to consider the validity of a version of parabolic Harnack inequality on a general complete Riemannian manifold. We shall show the validity of such a parabolic Harnack inequality is equivalent to the existence of Gaussian upper and lower bounds on the heat kernel. Previously, Saloff-Coste [SC] and Grigor'yan [G] had obtained another type of characterization for the parabolic Harnack inequality. To recall their results, let us first introduce some definitions.

Definition 4.1. Let $(M, g)$ be a complete Riemannian manifold. Then $(M, g)$ is said to satisfy the parabolic Harnack inequality (PHI) on balls of radius $r_{0}$ if there exists a constant $C$ depending only on the parameters $0<\varepsilon<\eta<\delta<1$, such that, for any $x \in M$, and real $s$, and any $0<r<r_{0}$, any nonnegative solution $u$ of $\left(\Delta-\frac{\partial}{\partial t}\right) u=0$ in $Q=\left(s-r^{2}, s\right) \times B_{x}(r)$ satisfies

$$
\sup _{Q_{-}}\{u\} \leq C \inf _{Q_{+}}\{u\}
$$

where

$$
Q_{-}=\left[s-\delta r^{2}, s-\eta r^{2}\right] \times B_{x}(\delta r)
$$

and

$$
Q_{+}=\left[s-\varepsilon r^{2}, s\right) \times B_{x}(\delta r)
$$

Definition 4.2. $\quad M$ satisfies volume doubling property on balls of radius $r_{0}$ if there exists constant $C$ such that

$$
V_{x}(2 r) \leq C V_{x}(r) \text { for any } 0<r<r_{0} \text { and } x \in M,
$$

where $V_{x}(r)$ denotes the volume of the geodesic ball $B_{x}(r)$ in $M$.

Definition 4.3. $\quad M$ satisfies weak-Neumann Poincaré inequality on balls of radius $r_{0}$ if there exists a constant $C$ such that

$$
\inf _{\alpha \in \mathbf{R}} \int_{B_{x}(r)}|f-\alpha|^{2} d \mu \leq C r^{2} \int_{B_{x}(2 r)}|\nabla f|^{2} d \mu
$$


for any $0<r<r_{0}, x \in M$ and $f \in C^{\infty}(M)$.

The following theorem is a special form of the more general results proved independently by Saloff-Coste [SC] and Grigor'yan [G].

\section{Theorem 4.4.}

(a) $M$ satisfies (PHI) on balls of radius $r_{0}$ if $M$ satisfies both volume doubling property and weak-Neumann Poincaré inequality on balls of radius $4 r_{0}$.

(b) $M$ satisfies both volume doubling property and weak-Neumann Poincaré inequality on balls of radius $r_{0}$ if $M$ satisfies (PHI) on balls of radius $2 r_{0}$.

For a general complete manifold, it is well-known that there exists a minimal heat kernel $H(x, y, t)$. We have the following result.

\section{Theorem 4.5.}

(a) If $M$ satisfies (PHI) on balls of radius $r_{0}$, then there exist some constants $C_{1}, C_{2}, C_{3}$ and $C_{4}$ such that

$$
\begin{aligned}
C_{1} V_{x}^{-1}(\sqrt{t}) \exp \left(-\frac{r^{2}(x, y)}{C_{2} t}\right) & \leq H(x, y, t) \\
& \leq C_{3} V_{x}^{-1}(\sqrt{t}) \exp \left(-\frac{r^{2}(x, y)}{C_{4} t}\right)
\end{aligned}
$$

for any $x, y$ and $t$ such that $r(x, y) \leq r_{1}<\frac{r_{0}}{2}$ and $t<r_{1}^{2}$, where $r(x, y)$ denotes the distance between $x$ and $y$.

(b) If there exist some constants $C_{1}, C_{2}, C_{3}$ and $C_{4}$ such that

$$
\begin{aligned}
C_{1} V_{x}^{-1}(\sqrt{t}) \exp \left(-\frac{r^{2}(x, y)}{C_{2} t}\right) & \leq H(x, y, t) \\
& \leq C_{3} V_{x}^{-1}(\sqrt{t}) \exp \left(-\frac{r^{2}(x, y)}{C_{4} t}\right)
\end{aligned}
$$

for any $x, y$ and $t$ such that $r(x, y) \leq r_{0}$ and $t \leq r_{0}^{2}$, then $M$ satisfies (PHI) on balls of radius $r_{1}$ for any $r_{1}<\frac{r_{0}}{8}$.

Corollary 4.6. $M$ satisfies (PHI) if and only if there exist some constants $C_{1}, C_{2}, C_{3}$ and $C_{4}$ such that

$$
C_{1} V_{x}^{-1}(\sqrt{t}) \exp \left(-\frac{r^{2}(x, y)}{C_{2} t}\right) \leq H(x, y, t) \leq C_{3} V_{x}^{-1}(\sqrt{t}) \exp \left(-\frac{r^{2}(x, y)}{C_{4} t}\right)
$$


for any $(x, y, t) \in M \times M \times(0, \infty)$, where $r(x, y)$ denotes the distance between $x$ and $y$.

Proof of Theorem 4.5. We first show that the validity of (PHI) on balls of radius $r_{0}$ implies the heat kernel bounds. Since by Theorem 4.4, $M$ satisfies volume doubling property on balls of radius $\frac{r_{0}}{2}$, the argument of $\mathrm{P}$. Li and S.T. Yau $[\mathbf{L}-\mathbf{Y}]$ then implies

$$
H(x, y, t) \leq C_{3} V_{x}^{-1}(\sqrt{t}) \exp \left(-\frac{r^{2}(x, y)}{C_{4} t}\right)
$$

for any $x, y$ and $t$ such that $r(x, y) \leq r_{1}<\frac{r_{0}}{2}$ and $t<r_{1}^{2}$, where $C_{3}$ and $C_{4}$ are constants depending on $r_{1}$. To establish the desired lower bound of $H(x, y, t)$, for any $x \in M$ and $r \leq r_{1}<r_{0}$, we consider the function defined by $u(z, s)=\int_{B_{x}(r)} H(y, z, s) d y$ when $s>0$ and $u(z, s)=1$ when $s \leq 0$. This function is a nonnegative solution of $\left(\triangle-\frac{\partial}{\partial t}\right) u=0$ on $B_{x}(r) \times(-\infty,+\infty)$. Hence we have

$$
\begin{aligned}
1=u\left(x,-r^{2} / 4\right) & \leq C u\left(x, r^{2} / 2\right) \\
& =C \int_{B_{x}(r)} H\left(y, x, r^{2} / 2\right) d y \leq C^{2} V_{x}(r) H\left(x, x, r^{2}\right) .
\end{aligned}
$$

Therefore,

$$
H\left(x, x, r^{2}\right) \geq C V_{x}^{-1}(r) .
$$

Using the Harnack inequality and the volume doubling property, one easily sees from (4.13) that

$$
H(x, y, t) \geq C V_{x}^{-1}(\sqrt{t}) \text { for } r(x, y) \leq \sqrt{t} \leq r_{1}<r_{0} \text { and } t>0,
$$

where $C$ is a constant.

For arbitrary $x, y \in M$ with $r(x, y) \leq r_{1}<r_{0} / 2$ and $t \leq r_{1}^{2}$, there exists a positive integer $N \geq 1$ such that $N-1<\frac{4 r^{2}(x, y)}{t} \leq N$. Let $\gamma(t)$ be a minimal geodesic connecting $x$ and $y$ which is parametrized by the arclength. Let $q_{i}=\gamma\left(\frac{\operatorname{ir}(x, y)}{N}\right), i=0, \ldots, N$. Then by the semi-group property of $H(x, y, t)$, we get 


$$
\begin{aligned}
& =\int_{M} \ldots \int_{M} H\left(x, z_{1}, \frac{t}{N}\right) H\left(z_{1}, z_{2}, \frac{t}{N}\right) \ldots H\left(z_{N-1}, y, \frac{t}{N}\right) d z_{1} \ldots d z_{N-1} \\
& \geq \int_{B_{q_{N-1}}\left(\frac{r}{N}\right)} \ldots \int_{B_{q_{1}}\left(\frac{r}{N}\right)} H\left(x, z_{1}, \frac{t}{N}\right) \ldots H\left(z_{N-1}, y, \frac{t}{N}\right) d z_{1} \ldots d z_{N-1} .
\end{aligned}
$$

For $z_{1} \in B_{q_{1}}\left(\frac{r}{N}\right), r\left(x, z_{1}\right) \leq r\left(x, q_{1}\right)+r\left(q_{1}, z_{1}\right) \leq \frac{2 r}{N}$. But $\frac{4 r^{2}}{t} \leq N$.

Therefore $r\left(x, z_{1}\right) \leq \sqrt{\frac{t}{N}}$ and by (4.14) $H\left(x, z_{1}, \frac{t}{N}\right) \geq C V_{x}^{-1}\left(\sqrt{\frac{t}{N}}\right)$.

Similarly, for $z_{i-1} \in B_{q_{i-1}}\left(\frac{r}{N}\right)$ and $z_{i} \in B_{q_{i}}\left(\frac{r}{N}\right)$, we have

$$
H\left(z_{i-1}, z_{i}, \frac{t}{N}\right) \geq C V_{q_{i-1}}^{-1}\left(2 \sqrt{\frac{t}{N}}\right), \quad i=2, \ldots, N-1 .
$$

Hence by (4.15),

$$
\begin{aligned}
H(x, y, t) \geq & C^{N-1} V_{x}^{-1}\left(\sqrt{\frac{t}{N}}\right) V_{q_{1}}^{-1}\left(2 \sqrt{\frac{t}{N}}\right) \ldots V_{q_{N-1}}^{-1}\left(2 \sqrt{\frac{t}{N}}\right) \\
& \cdot V_{q_{1}}\left(\frac{r}{N}\right) \ldots V_{q_{N-1}}\left(\frac{r}{N}\right) .
\end{aligned}
$$

By the volume doubling property,

$$
\frac{V_{q_{i}}\left(\frac{r}{N}\right)}{V_{q_{i}}\left(2 \sqrt{\frac{t}{N}}\right)} \geq \frac{V_{q_{i}}\left(\frac{r}{N}\right)}{V_{q_{i}}\left(\frac{4 r}{N}\right)} \geq C_{5}, \quad \text { a constant. }
$$

Putting into (4.16), one concludes that

$$
\begin{aligned}
H(x, y, t) & \geq C^{N-1} C_{5}^{N-1} V_{x}^{-1}\left(\sqrt{\frac{t}{N}}\right) \\
& \geq C_{6}^{N-1} V_{x}^{-1}(\sqrt{t}) \\
& \geq C_{7} V_{x}^{-1}(\sqrt{t}) \exp \left(-C_{8} N\right) \\
& \geq C_{9} V_{x}^{-1}(\sqrt{t}) \exp \left(-\frac{r^{2}(x, y)}{C_{10} t}\right) .
\end{aligned}
$$

Therefore part (a) of the theorem is proved.

Now we come to prove part (b) of Theorem 4.5. In view of Theorem 4.4, we need only to check that $M$ satisfies volume doubling property and 
weak-Neumann Poincaré inequality on balls of radius $r_{0} / 2$. For any $x$ and $t \leq r_{0}^{2} / 2$, by the semi-group property of $H(x, y, t)$,

$$
\begin{aligned}
C_{3} V_{x}^{-1}(\sqrt{2 t}) & \geq H(x, x, 2 t)=\int_{M} H^{2}(x, y, t) d y \\
& \geq \int_{B_{x}(\sqrt{t})} C_{1}^{2} V_{x}^{-2}(\sqrt{t}) \exp \left(-\frac{1}{C_{2}}\right) d y \\
& \geq C V_{x}^{-1}(\sqrt{t}) \quad \text { for some } C>0 .
\end{aligned}
$$

Thus $V_{x}(\sqrt{2 t}) \leq \tilde{C} V_{x}(\sqrt{t})$ for all $0<t<r_{0} / \sqrt{2}$, where $\tilde{C}$ is a constant. Therefore $V_{x}(2 r) \leq \tilde{C} V_{x}(\sqrt{2} r) \leq \tilde{C}^{2} V_{x}(r)$ for all $r \leq r_{0} / 2$, and $M$ satisfies volume doubling property on balls of radius $r_{0} / 2$.

To show that $M$ satisfies weak-Neumann Poincaré inequality on balls of radius $r_{0} / 2$, we first establish the following claim.

Claim. For any $p \in M$ and $R \leq r_{0} / 2$, there exist constants $C_{1}, C_{2}$ and $C_{3}$ independent of $p$ and $R$ such that

$$
H_{R}\left(x, y, C_{1} R^{2}\right) \geq C_{3} V_{p}^{-1}(R)
$$

for all $x$ and $y$ satisfying

$$
r(p, x) \leq C_{2} R, \quad r(p, y) \leq C_{2} R,
$$

where $H_{R}(x, y, t)$ is the heat kernel of $B_{p}(R)$ satisfying the Dirichlet boundary condition.

Once the claim is established, then by an argument of Fabes and Stroock [F-S], one concludes that $M$ satisfies the weak-Neumann Poincaré inequality on balls of radius $r_{0} / 2$.

To check the claim, by the maximum principle, we have

$$
H_{R}(x, y, t) \geq H(x, y, t)-\max _{\substack{0 \leq s \leq t \\ z \in S_{p}(R)}} H(x, z, s) .
$$

From the assumption, there exists a constant $C$ such that for $t \leq r_{0}^{2}$,

$$
\max _{\substack{0 \leq s \leq t \\ z \in S_{p}(R)}} H(x, z, s) \leq \max _{0 \leq s \leq t} C V_{x}^{-1}(\sqrt{s}) \exp \left(-\frac{R^{2}}{C s}\right)
$$

for all $x$ such that $r(p, x) \leq C_{2} R, C_{2} \leq \frac{1}{2}$. Thus

$$
\max _{\substack{0 \leq s \leq t \\ z \in S_{p}(R)}} H(x, z, s) \leq C V_{x}^{-1}(\sqrt{t}) \max _{0 \leq s \leq t} \frac{V_{x}(\sqrt{t})}{V_{x}(\sqrt{s})} \exp \left(-\frac{R^{2}}{C s}\right) .
$$


Since $M$ satisfies volume doubling property on balls of radius $r_{0} / 2$, for some constant $\alpha>0$ and constant $C>0, \frac{V_{x}(\sqrt{t})}{V_{x}(\sqrt{s})} \leq C\left(\frac{\sqrt{t}}{\sqrt{s}}\right)^{\alpha}$ (see [G]) for all $\sqrt{s} \leq \sqrt{t} \leq r_{0} / 2$. In particular, (4.17) implies that

$$
\begin{aligned}
& \max _{\substack{0 \leq s \leq t \\
z \in S_{p}(R)}} H(x, z, s) \\
& \leq C V_{x}^{-1}(\sqrt{t}) \max _{0 \leq s \leq t}\left(\frac{t}{s}\right)^{\alpha} \exp \left(-\frac{R^{2}}{C s}\right) \\
& \leq C_{3} V_{x}^{-1}(\sqrt{t}) \exp \left(-\frac{R^{2}}{C_{4} t}\right)
\end{aligned}
$$

if $t \leq C_{5} R^{2}$ for some $C_{5}>0$. Thus, for all $t \leq C_{5} R^{2}$,

$$
\begin{aligned}
& H_{R}(x, y, t) \\
& \geq C_{1} V_{x}^{-1}(\sqrt{t}) \exp \left(-\frac{r^{2}(x, y)}{C_{2} t}\right)-C_{3} V_{x}^{-1}(\sqrt{t}) \exp \left(-\frac{R^{2}}{C_{4} t}\right) .
\end{aligned}
$$

If we choose $C_{6}$ sufficiently small such that

$$
r(p, x) \leq C_{6} R \quad \text { and } \quad r(p, y) \leq C_{6} R,
$$

then $r(x, y) \leq 2 C_{6} R$ and

$$
C_{3} \exp \left(-\frac{R^{2}}{C_{4} t}\right) \leq \frac{1}{2} C_{1} \exp \left(-\frac{r^{2}(x, y)}{C_{2} t}\right) \quad \text { for all } t \leq C_{5} R^{2}
$$

Together (4.18) and (4.19), we have

$$
H_{R}(x, y, t) \geq C V_{x}^{-1}(\sqrt{t}) \exp \left(-\frac{r^{2}(x, y)}{C t}\right)
$$

for some $C>0$ and all $t \leq C_{5} R^{2}$, where $r(p, x) \leq C_{6} R$ and $r(p, y) \leq C_{6} R$. But

$$
V_{x}^{-1}(\sqrt{t}) \geq V_{p}^{-1}(\sqrt{t}+r(p, x)) \geq V_{p}^{-1}(2 R) \geq C V_{p}^{-1}(R)
$$

by the volume doubling property. Therefore

$$
H_{R}(x, y, t) \geq C V_{p}^{-1}(R) \exp \left(-\frac{r^{2}(x, y)}{C t}\right)
$$

for $t \leq C_{5} R^{2}$ and $r(p, x) \leq C_{6} R, r(p, y) \leq C_{6} R$. In particular, let $t=C_{5} R^{2}$ in (4.20), the claim follows. This completes our proof of Theorem 4.5. 


\section{References}

[C] R. Chen, Neumann eigenvalue estimate on a compact Riemannian manifold, Proc. AMS, 108 (1990), 961-970.

[C-L] S.Y. Cheng and P. Li, Heat kernel estimates and lower bounds of eigenvalues, Comment. Math. Helv., 56 (1981), 327-338.

[Cr] C.B. Croke, Some isoperimetric inequalities and eigenvalue estimates, Ann. Scien. Ec. Norm. Sup., 13 (1980), 419-435.

[F-S] E. Fabes and D.W. Stroock, A new proof of Moser's parabolic Harnack inequality using the old ideas of Nash, Arch. Rational. Mech. Anal., 96 (1986), 327-338.

[G] A.A. Grigor'yan, The heat equation on noncompact Riemannian manifolds, Math. USSR Sbornik, 72 (1992), 47-77.

[L] P. Li, Lecture Notes on heat kernels, UC, Irvine, 1991.

[L-Y] P. Li and S.T. Yau, On the parabolic heat kernel of the Schrödinger operator, Acta Math., 156 (1986), 153-201.

[SC] L. Saloff-Coste, A note on Poincaré, Sobolev, and Harnack inequalities, Int. Math. Research Notices, 2 (1992), 27-38.

[V] N. Varopoulos, Hardy-Littlewood theory for semigroups, J. Funct. Anal., 98 (1985), 240-260.

Received April 21, 1995.

STANFORD UNIVERSITY

StANFORD, CA 94305

E-mail address: jwang@math.stanford.edu 